\title{
A NONCOMMUTATIVE CYCLE INDEX AND NEW BASES OF QUASI-SYMMETRIC FUNCTIONS AND NONCOMMUTATIVE SYMMETRIC FUNCTIONS
}

\author{
JEAN-CHRISTOPHE NOVELLI, JEAN-YVES THIBON, AND FRÉDÉRIC TOUMAZET
}

\begin{abstract}
We define a new basis of the algebra of quasi-symmetric functions by lifting the cycle-index polynomials of symmetric groups to noncommutative polynomials with coefficients in the algebra of free quasi-symmetric functions, and then projecting the coefficients to QSym. By duality, we obtain a basis of noncommutative symmetric functions, for which a product formula and a recurrence in the form of a combinatorial complex are obtained. This basis allows to identify noncommutative symmetric functions with the quotient of FQSym induced by the pattern-replacement relation $321 \equiv 231$ and $312 \equiv 132$.
\end{abstract}

\section{INTRODUCTION}

For a partition $\mu=1^{m_{1}} 2^{m_{2}} \cdots n^{m_{n}}$ of $n$, the coefficient of $p_{\mu}=p_{1}^{m_{1}} p_{2}^{m_{2}} \cdots p_{n}^{m_{n}}$ in the polynomial $h_{n}\left(p_{1}, \ldots, p_{n}\right)$ defined by $h_{0}=1$ and the recursion

$$
n h_{n}=h_{n-1} p_{1}+h_{n-2} p_{2}+\cdots+p_{n}
$$

is the number of permutations of cycle type $\mu$, divided by $n$ !. Thus, $h_{n}$ is the cycle index of the symmetric group $\mathfrak{S}_{n}$, and if the $p_{k}$ are interpreted as the power sums symmetric functions, then $h_{n}$ is the complete symmetric function (sum of all monomials of degree $n$ ), cf. [19].

There is a noncommutative analogue of this polynomial, which defines the so-called noncommutative power sums of the first kind $\Psi_{n}$ in terms of the noncommutative complete functions $S_{n}$ [6]

$$
n S_{n}=S_{n-1} \Psi_{1}+S_{n-2} \Psi_{2}+\cdots+\Psi_{n} .
$$

It is not difficult to see that the coefficient $c_{I}$ in

$$
n ! S_{n}=\sum_{I} c_{I} \Psi^{I}=\sum_{I} \frac{n !}{i_{1}\left(i_{1}+i_{2}\right) \cdots\left(i_{1}+i_{2}+\cdots i_{r}\right)} \Psi^{I}
$$

counts the permutations with ordered cycle type $I$, that is, the composition consisting of the lengths of the cycles, ordered by increasing values of their maxima.

Date: March 27, 2019.

1991 Mathematics Subject Classification. 16T30,05E05,05A18.

Key words and phrases. Noncommutative symmetric functions, Quasi-symmetric functions, Dendriform algebras. 
For example,

$$
\begin{aligned}
& 4 ! h_{4}=p_{1111}+6 p_{211}+3 p_{22}+8 p_{31}+6 p_{4}, \\
& 4 ! S_{4}=\Psi^{1111}+3 \Psi^{112}+2 \Psi^{121}+6 \Psi^{13}+\Psi^{211}+3 \Psi^{22}+2 \Psi^{31}+6 \Psi_{4},
\end{aligned}
$$

the 3 transpositions of ordered cycle type $(1,1,2)$ being $(1)(2)(43),(1)(3)(42)$ and $(2)(3)(41)$, while $(1)(32)(4)$ and $(2)(31)(4)$ are of type $(1,2,1)$, and $(21)(3)(4)$ is of type $(2,1,1)$.

Setting $y_{n}=(n-1) ! p_{n}$ and $Y_{n}=(n-1) ! \Psi_{n}$, which amounts to forgetting the order inside the cycles, $n ! h_{n}$ and $n ! S_{n}$ become respectively the usual exponential Bell polynomial $B_{n}\left(y_{1}, \ldots, y_{n}\right)$, whose coefficients count set partitions according to the sizes of the blocks, and its natural noncommutative analogue [24] refining this counting according to a canonical ordering of the blocks.

This situation has been exploited is [20] in order to define further generalizations of the Bell polynomials, whose coefficients live in the algebra of free quasi-symmetric functions and encode the set partitions counted by the original coefficients in a way preserving their algebraic properties. The commutative images of these coefficients are quasi-symmetric functions, which turn out to form the dual immaculate basis of [1] up to reversal of the compositions.

The aim of this note is to apply the same strategy to the noncommutative cycle index with its original variables, that is, we keep $Y_{n}=\Psi_{n}$, and define successively a $q$ analogue $c_{I}(q)$ of $c_{I}$, a free quasi-symmetric function $\mathbf{C}_{I}$, a quasi-symmetric function $C_{I}$, and finally, a basis $U_{I}=C_{\bar{I}^{\sim}}$ of $Q S y m$, and its dual basis $V_{I}$ for noncommutative symmetric functions.

This paper is a continuation of [20], to which the reader may refer for background and notation.

\section{The Saillances statistics, And a $q$-Analogue}

As the ordered cycle type appears to be of little significance from a group theoretical point of view, it can be advantageously replaced by a natural statistic having the same distribution.

Following [18, Chap. 10], let us say that a word $w=a_{1} a_{2} \cdots a_{n}$ over the integers is initially dominated if $a_{1}>a_{2}, \ldots, a_{n}$. Order these words with respect to their maximal letter. Then, a permutation has a unique increasing factorisation

$$
\sigma=u_{1} u_{2} \cdots u_{r}
$$

into initially dominated words. The composition

$$
\mathrm{SC}(\sigma)=\left(\left|u_{1}\right|,\left|u_{2}\right|, \ldots,\left|u_{r}\right|\right)
$$

will be called the saillance composition of $\sigma$.

For example, for $\sigma=351274698$, the factorisation is $3 \cdot 512 \cdot 746 \cdot 98$, and $\operatorname{SC}(\sigma)=$ $(1,3,3,2)$.

The natural $q$-analogue of $\Psi_{n}$ is [10]

$$
\Theta_{n}(q)=\frac{S_{n}((1-q) A)}{1-q}=\sum_{k=0}^{n-1}(-q)^{k} R_{1^{k}, n-k}
$$


and the expression

$$
[n]_{q} ! S_{n}(A)=\sum_{I} c_{I}(q) \Theta^{I}(q)=\sum_{I} q^{\operatorname{maj}(I)} \frac{[n]_{q} !}{\left[i_{1}\right]_{q}\left[i_{1}+i_{2}\right]_{q} \cdots\left[i_{1}+i_{2}+\cdots i_{r}\right]_{q}} \Theta^{I}(q)
$$

provides immediately a $q$-analogue of $c_{I}$ :

Proposition 2.1. The polynomial $c_{I}(q)$ counts the permutations of saillance composition I by number of non-inversions:

$$
c_{I}(q)=\sum_{\operatorname{SC}(\sigma)=I} q^{\left(\begin{array}{c}
n \\
2
\end{array}\right)-\operatorname{inv}(\sigma)} .
$$

To prove this, we first observe that the inverses of the permutations having a given saillance composition are the linear extensions of a poset, whose Hasse diagram is a tree of a very special shape (see Figure 1).

Indeed, the conditions $\sigma_{1}>\sigma_{2}, \ldots, \sigma_{i_{1}}, \sigma_{1}<\sigma_{i_{1}+1}>\sigma_{i_{1}+2}, \ldots, \sigma_{i_{1}+i_{2}}$, etc., translate into the conditions that in the inverse permutation, 1 must be to the right of $2,3, \ldots, i_{1}$, and that $i_{1}+1$ must be to the right of $i_{1}+2, \ldots, i_{1}+i_{2}$ and of $1,2, \ldots i_{1}$, and so on. This defines a poset $P(I)$, where $i<_{P} j$ iff $j$ must be to the right of $i$ in $\sigma^{-1}$.

For example, for $I=(2,3,2), 2<_{P} 1 ; 1<_{P} 3>_{P} 4,5 ; 3<_{P} 6>_{P} 7$, which gives the comb-tree of Figure 1 .

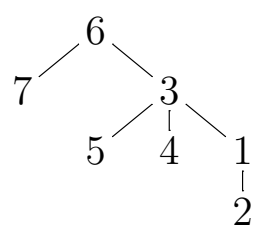

Figure 1. The poset $P(2,3,2)$.

Thus, we can apply the Björner-Wachs $q$-hook-length formula [2] to count the linear extensions of this poset by number of inversions. Since this is clearly a recursive labeling, this gives

$$
q^{\operatorname{inv}\left(\alpha_{I}\right)} \frac{[n]_{q} !}{\left[i_{1}\right]_{q}\left[i_{1}+i_{2}\right]_{q} \cdots\left[i_{1}+i_{2}+\cdots i_{r}\right]_{q}}
$$

where

$$
\alpha_{I}=2 \cdots i_{1} 1 \cdot\left(i_{1}+2\right) \cdots\left(i_{1}+i_{2}\right) \cdot\left(i_{1}+1\right) \cdots
$$

is the minimal linear extension, whose number of inversions is $\sum_{k}\left(i_{k}-1\right)=n-\ell(I)$.

Thus,

$$
\begin{aligned}
\sum_{\operatorname{SC}(\sigma)=I} q^{\operatorname{inv}(\sigma)} & =q^{n-\ell(I)} \frac{[n]_{q} !}{\left[i_{1}\right]_{q}\left[i_{1}+i_{2}\right]_{q} \cdots\left[i_{1}+i_{2}+\cdots i_{r}\right]_{q}} \\
& =q^{\left(\begin{array}{l}
n \\
2
\end{array}\right)} c_{I}\left(q^{-1}\right)
\end{aligned}
$$


(since $\left.q^{\operatorname{maj}(I)}=q^{i_{1}} q^{i_{1}+i_{2}} \cdots q^{i_{1}+\cdots+i_{r-1}}\right)$, so that

$$
c_{I}(q)=\sum_{\operatorname{SC}(\sigma)=I} q^{\left(\begin{array}{l}
n \\
2
\end{array}\right)-\operatorname{inv}(\sigma)} .
$$

For $P(2,3,2)$ this polynomial is

$$
q^{17}+3 q^{16}+6 q^{15}+9 q^{14}+11 q^{13}+12 q^{12}+11 q^{11}+9 q^{10}+6 q^{9}+3 q^{8}+q^{7} .
$$

Alternatively, we may replace $\Theta_{n}(q)$ by $\tilde{\Theta}_{n}(q):=(q-1)^{-1} S_{n}((q-1) A)$. Then, the coefficient $\tilde{c}_{I}(q)$ in

$$
[n]_{q} ! S_{n}(A)=\sum_{I} \tilde{c}_{I}(q) \tilde{\Theta}^{I}(q)=\sum_{I} \frac{[n]_{q} !}{\left[i_{1}\right]_{q}\left[i_{1}+i_{2}\right]_{q} \cdots\left[i_{1}+i_{2}+\cdots i_{r}\right]_{q}} \tilde{\Theta}^{I}(q)
$$

counts permutations of ordered cycle type $I$ by Carlitz's statistic [3], [25, A129178]

$$
\operatorname{invc}(\sigma)=\operatorname{inv}\left(f_{1}(\sigma)\right)
$$

where $f_{1}$ is Foata's first fundamental transformation: express $\sigma$ in standard cycle form (i.e., cycles ordered by increasing smallest elements with each cycle written with its smallest element in the first position), then remove the parentheses [18].

\section{A DENDRIFORM EXPONENTIAL}

We can now define in FQSym, as in [20] for the Bell polynomials,

$$
\mathbf{C}_{I}:=\sum_{\mathrm{SC}(\sigma)=I} \mathbf{G}_{\sigma}
$$

and investigate the commutative image of these elements in QSym.

The $\mathbf{C}_{I}$ can be expressed in terms of the dendriform operations of FQSym. We have

$$
\mathbf{C}_{n}=\mathbf{G}_{1} \prec \mathbf{G}_{1}^{n-1}
$$

and

$$
\mathbf{C}_{I}=\left(\cdots\left(\mathbf{C}_{i_{1}} \succ \mathbf{C}_{i_{2}}\right) \succ \cdots\right) \succ \mathbf{C}_{i_{r}}
$$

Their generating series in FQSym $\otimes \mathbb{K}\left\langle Y_{1}, Y_{2}, \cdots\right\rangle$

$$
Z:=\sum_{I} \mathbf{C}_{I} Y^{I}
$$

satisfies therefore

$$
Z=1+Z \succ C, \text { with } C:=\sum_{n \geq 1} \mathbf{C}_{n} Y_{n} .
$$

Thus, $\mathbf{C}_{I}$ belongs to the Loday-Ronco algebra PBT: it is the sum of all $\mathbf{P}_{T}$ where $T$ has a left branch with $r=\ell(I)$ nodes, on which are grafted as right subtrees all binary trees of sizes $i_{1}-1, i_{2}-1, \ldots, i_{r}-1$, starting from the bottom. 


$$
\begin{aligned}
Z_{1} & =\mathbf{G}_{1} Y_{1} \\
Z_{2} & =\mathbf{G}_{12} Y^{11}+\mathbf{G}_{21} Y_{2} \\
Z_{3} & =\mathbf{G}_{123} Y^{111}+\left(\mathbf{G}_{132}+\mathbf{G}_{231}\right) Y^{12}+\mathbf{G}_{213} Y^{21}+\left(\mathbf{G}_{312}+\mathbf{G}_{321}\right) Y_{3} \\
Z_{4} & =\mathbf{G}_{1234} Y^{1111}+\left(\mathbf{G}_{1243}+\mathbf{G}_{1342}+\mathbf{G}_{2341}\right) Y^{112}+\left(\mathbf{G}_{1324}+\mathbf{G}_{2314}\right) Y^{121}+\mathbf{G}_{2134} Y^{211} \\
& +\left(\mathbf{G}_{1423}+\mathbf{G}_{2413}+\mathbf{G}_{3412}+\mathbf{G}_{1432}+\mathbf{G}_{2431}+\mathbf{G}_{3421}\right) Y^{13}+\left(\mathbf{G}_{2143}+\mathbf{G}_{3241}+\mathbf{G}_{3142}\right) Y^{22} \\
& +\left(\mathbf{G}_{4123}+\mathbf{G}_{4132}+\mathbf{G}_{4213}+\mathbf{G}_{4231}+\mathbf{G}_{4312}+\mathbf{G}_{4321}\right) Y_{4}
\end{aligned}
$$

FiguRe 2. Values of $Z_{n}$ for $n \leq 4$.

Proposition 3.1. The $\mathbf{C}_{I}$ span a sub-coalgebra of $\mathbf{F Q S y m}$, and the coefficients $a_{I}^{J K}$ in

$$
\Delta \mathbf{C}_{I}=\sum_{J K} a_{I}^{J K} \mathbf{C}_{J} \otimes \mathbf{C}_{K}
$$

are nonnegative integers.

Proof - Since FQSym is self-dual,we can write

$$
\begin{aligned}
\Delta \mathbf{C}_{I} & =\sum_{\alpha, \beta}\left\langle\Delta \mathbf{C}_{I}, \mathbf{F}_{\alpha} \otimes \mathbf{F}_{\beta}\right\rangle \mathbf{G}_{\alpha} \otimes \mathbf{G}_{\beta} \\
& =\sum_{\alpha, \beta}\left\langle\mathbf{C}_{I}, \mathbf{F}_{\alpha} \mathbf{F}_{\beta}\right\rangle \mathbf{G}_{\alpha} \otimes \mathbf{G}_{\beta} \\
& =\sum_{\operatorname{SC}(\gamma)=I} \sum_{\alpha, \beta}\left\langle\mathbf{G}_{\gamma}, \mathbf{F}_{\alpha} \mathbf{F}_{\beta}\right\rangle \mathbf{G}_{\alpha} \otimes \mathbf{G}_{\beta},
\end{aligned}
$$

and it is clear that the distribution of the saillances in the shifted shuffle $\mathbf{F}_{\alpha} \mathbf{F}_{\beta}$ depends only on $\operatorname{SC}(\alpha)$ and $\operatorname{SC}(\beta)$. Thus (24) holds, and $a_{I}^{J K}$ is the number of triple of permutations $(\alpha, \beta, \gamma)$ such that $\operatorname{SC}(\alpha)=J, \operatorname{SC}(\beta)=K, \operatorname{SC}(\gamma)=I$, and $\gamma$ occurs in the shifted shuffle of $\alpha$ and $\beta$.

A closed formula for $a_{I}^{J K}$ will be given in the sequel.

Note that $Z$ is an exponential without denominators according to the definition of [17] $\left(Z=e^{\prime}(C)\right.$ in the notation of this reference), and that $\sum_{n} \mathbf{C}_{n}=-L\left(-\mathbf{G}_{1}\right)$ is a logarithm without denominators.

\section{A BASIS OF QSYm}

We can now take the commutative images of the $\mathbf{C}_{I}$, and investigate the resulting quasi-symmetric functions.

It turns out that $\mathbf{C}_{I}(X)$ does not always contain $F_{I}$, but always contains $\bar{I}^{\sim}$. Thus, we define

$$
U_{I}(X)=\sum_{\mathrm{SC}(\sigma)=I^{\sim}} F_{\mathrm{RC}(\sigma)}
$$


where $\mathrm{RC}(\sigma)$ is the recoil composition of $\sigma$.

The first values are tabulated on Figure 3. This table and all tables afterwards are quite large and were put at the end of the paper.

Proposition 4.1. The transition matrix $M_{n}$, whose column indexed by $J$ gives the coefficients of $U_{J}$ on the $F_{I}$ is upper unitriangular when compositions are ordered lexicographically by length, see Figure 4.

Proof - Consider a permutation such that $\mathrm{SC}(\sigma)=I$. Then the values in position 1 , $i_{1}+1, \ldots, i_{1}+\cdots+i_{r-1}+1$ cannot be recoils since they have no greater value to their left. Hence, the total number of recoils of $\sigma$ cannot be greater than $|I|-l(I)$ so that the length of the recoil composition of $\sigma$ cannot be greater than the length of $\bar{I}^{\sim}$. If the length of the recoil composition of $\sigma$ is strictly smaller than the length of $\bar{I}^{\sim}$, this composition appears before $\bar{I}^{\sim}$ in our order. Otherwise, all positions except the above mentioned ones are recoils, so that, given that $\sigma_{1}$ is greater than $\sigma_{k}$ for all $k \in\left[2, i_{1}\right]$, the smallest possible non-recoil of $\sigma$ is at least $i_{1}$. This means that all values smaller than $i_{1}$ are recoils of $\sigma$. Now, if $i_{1}$ is not a recoil, the recoil composition of $\sigma$ is strictly smaller than $\bar{I}^{\sim}$. Otherwise, they have same first part and one can conclude by induction on the number of parts of $I$ (the next smallest possible recoil is then $i_{1}+i_{2}$, and so on).

For example, for $I=(2,3,1,2)$, values at positions $1,3,6$, and 7 cannot be recoils so that the total number of recoils cannot be greater than $8-4=4$, which is the number of recoils of $\bar{I}^{\sim}=(1,2,1,3,1)$. If there are exactly 4 recoils, hence at positions $2,5,4,8$, the smallest non-recoil value is 2 since $\sigma_{1}>\sigma_{2}$, and if 2 is a recoil, then the second smallest non-recoil is 5 since $\sigma_{3}>\sigma_{4}$ and $\sigma_{3}>\sigma_{5}$. If one computes the largest set of recoils (sorted by increasing length then by lexicographic order), it is exactly $\{2,5,6,8\}$, which is $\operatorname{Des}(I)$, so that the recoil composition of any $\sigma$ such that $\mathrm{SC}(\sigma)=I$ is at most (for the specified order on compositions) its complement $\{1,3,4,7\}=\operatorname{Des}\left(\bar{I}^{\sim}\right)$.

\section{The DUAL Basis in Sym}

Let $V_{I}$ denote the dual basis of $U_{I}$. Reading row $I$ of $M_{n}$, we have the expansion of the ribbon $R_{I}$ on the $V_{J}$, which is therefore nonnegative.

The first values of the $V_{I}$ on the ribbon basis are given on Figure 5 .

As we shall see, it is rather the expansion on the elementary basis $\Lambda^{I}$ which is relevant to the understanding of the $V_{I}$. The first values are given on Figure 6 ,

To investigate the multiplicative structure of the $V_{I}$, we first observe that Sym can be identified (as an algebra) with the quotient of FQSym by the ideal $\mathcal{J}$ generated by

$$
\left\{\mathbf{F}_{\sigma}-\mathbf{F}_{\tau} \mid \mathrm{SC}(\sigma)=\mathrm{SC}(\tau)\right\}
$$

Indeed, since the commutative image map $\pi:$ FQSym $\rightarrow$ QSym is an epimorphism of Hopf algebras, we have

$$
\Delta \mathbf{C}_{I}(X)=\sum_{J K} a_{I}^{J K} \mathbf{C}_{J}(X) \otimes \mathbf{C}_{K}(X)
$$


with the same coefficients $a_{I}^{J K}$ as in (24). Thus,

$$
\Delta U_{I}(X)=\sum_{J K} a_{\bar{I}^{\sim}}^{\bar{J}^{\sim}} U_{J}(X) \otimes U_{K}(X),
$$

and $V_{I}$ may be identified with the class $\overline{\mathbf{F}}_{\sigma}=\mathbf{F}_{\sigma} \bmod \mathcal{J}$ for $\operatorname{SC}(\sigma)=\bar{I}^{\sim}$.

Note that this construction is similar to that of Tevlin's fundamental basis given in [9. Moreover, the equivalence classes here can be described in terms of patternreplacement relations:

Proposition 5.1. Two permutations $\sigma, \tau \in \mathfrak{S}_{n}$ have the same saillance composition iff they are equivalent modulo the pattern replacement relations

$$
321 \equiv 231 \text { and } 312 \equiv 132 \text {. }
$$

These relations are the mirror images of those of [12, Section 3.11], see Section 6] for a proof of the proposition and further details about this equivalence.

The structure constants

$$
V_{I} V_{J}=\sum_{K} c_{I J}^{K} V_{K}
$$

are therefore given by the following rule. Take two permutations $\alpha$ with $S C(\alpha)=\bar{I}^{\sim}$, such that $\beta$ with $S C(\beta)=\bar{J}^{\sim}$. Then,

$$
c_{I J}^{K}=\#\left\{\mathbf{F}_{\gamma} \in \mathbf{F}_{\alpha} \mathbf{F}_{\beta} \mid S C(\gamma)=\bar{K}^{\sim}\right\} .
$$

This can be made more explicit. First, we can state a Pieri formula.

For a composition $I$ of $n$, denote by $I[k]$ the composition of $k$ whose ribbon diagram consists of the first $k$ boxes of that of $I$.

Proposition 5.2. Let $V_{I}^{\prime}=V_{\bar{I}^{\sim}}$. Then

$$
V_{I}^{\prime} \Lambda_{k}=\sum_{j=0}^{n}\left(\begin{array}{c}
k+j-1 \\
k-1
\end{array}\right) V_{I[n-j], k+j}^{\prime}
$$

Proof - For $\sigma \in \mathfrak{S}_{n}$, the saillance composition of a permutation $\tau$ occuring in the shifted shuffle of $\sigma$ with $\omega_{k}=k \cdots 21$ is determined by the position of $m=n+k$. The number of permutations in this shuffle for which $m$ is at position $n-j$ is $\left(\begin{array}{c}k+j-1 \\ k-1\end{array}\right)$.

For example,

$$
V_{32}^{\prime} \Lambda_{3}=\left(\begin{array}{l}
2 \\
2
\end{array}\right) V_{323}^{\prime}+\left(\begin{array}{l}
3 \\
2
\end{array}\right) V_{314}^{\prime}+\left(\begin{array}{l}
4 \\
2
\end{array}\right) V_{35}^{\prime}+\left(\begin{array}{l}
5 \\
2
\end{array}\right) V_{26}^{\prime}+\left(\begin{array}{l}
6 \\
2
\end{array}\right) V_{17}^{\prime}+\left(\begin{array}{l}
7 \\
2
\end{array}\right) V_{8}^{\prime}
$$

so that, complementing the compositions,

(38) $V_{1121} V_{111}=21 V_{11111111}+6 V_{1121111}+V_{112211}+3 V_{113111}+10 V_{1211111}+15 V_{2111111}$

Corollary 5.3. For $I=\left(i_{1}, \ldots, i_{r}\right)$,

$$
V_{I}^{\prime}=\sum_{k=i_{r}}^{n}(-1)^{k-i_{r}}\left(\begin{array}{c}
k-1 \\
i_{r}-1
\end{array}\right) V_{I[n-k]}^{\prime} V_{k}^{\prime}
$$


Proof - If we expand the products on the r.h.s. by the rule of Proposition 5.2, the coefficient of $V_{I[n-p], p}^{\prime}$ is

$$
\sum_{k=i_{r}}^{p}(-1)^{k}\left(\begin{array}{c}
k-1 \\
i_{r}-1
\end{array}\right)\left(\begin{array}{l}
p-1 \\
k-1
\end{array}\right)=(-1)^{i_{r}}\left(\begin{array}{c}
p-1 \\
i_{r}-1
\end{array}\right) \sum_{l=0}^{p-i_{r}}(-1)^{l}\left(\begin{array}{c}
p-i_{r} \\
l
\end{array}\right)=(-1)^{i_{r}} \delta_{p, i_{r}} .
$$

If we evaluate the alternating sum (39) step by step, we can observe that the partial sums are alternatively positive and negative. This suggests the existence of a combinatorial complex explaining the formula.

Lemma 5.4. Let $u, v$ be two words, and a be a letter. Then,

$$
u a v=\sum_{u_{1} u_{2}=u}(-1)^{\left|u_{2}\right|} u_{1} Ш\left(a\left(\overline{u_{2}} \amalg v\right)\right) .
$$

Proof - It is sufficient to prove the lemma for $u=12 \cdots k, a=k+1$, and $v=$ $k+2 \cdots n$. In this case, all terms, viewed as elements of FQSym in the F-basis, are noncommutative symmetric functions. The l.h.s. is $R_{n}$, and for $u_{1}=1 \cdots k-i$, the corresponding term of the sum is $(-1)^{i} R_{k-i} R_{1^{i}, n-k}$. Apart from the last one, which is $R_{1^{k}, n-k}$, each term is a sum of two ribbons, and two consecutive terms have exactly one ribbon in common.

For example, taking $k=2$, we can write

$$
1234=12 \varpi 34-1 ш 3(2 \amalg 4)+3(21 ш 4)
$$

which amounts to the identity

$$
R_{4}=R_{2} R_{2}-R_{1} R_{12}+R_{112}=\left(R_{4}+R_{22}\right)-\left(R_{22}+R_{112}\right)+R_{112} .
$$

Applying this to the permutation 2143, whose saillance composition is 22, we obtain

$$
2143=21 \amalg 43-2 \amalg 4(1 Ш 3)+4(12 \amalg 3)
$$

which translates into

$$
V_{22}^{\prime}=V_{2}^{\prime} V_{2}^{\prime}-2 V_{1}^{\prime} V_{3}^{\prime}+3 V_{4}^{\prime}
$$

Refining the argument of the proof of Proposition 5.2 yields the general product rule:

Theorem 5.5. The coefficients $\bar{c}_{I J}^{K}$ in the product

$$
V_{I}^{\prime} V_{J}^{\prime}=\sum_{K} \bar{c}_{I J}^{K} V_{K}^{\prime}
$$


where $\ell(I)=m, \ell(J)=p, \ell(K)=q$, and $r=q-p$, are given by

(47) $\bar{c}_{I J}^{K}=\left\{\begin{array}{l}\prod_{i=1}^{p}\left(\begin{array}{c}k_{i}-1 \\ j_{i}-1\end{array}\right) \text { if } q=p, \\ \prod_{i=1}^{p}\left(\begin{array}{c}k_{i+r}-1 \\ j_{i}-1\end{array}\right) \text { if } r>0,\left(k_{1}, \ldots ; k_{r-1}\right)=\left(i_{1}, \ldots, i_{r-1}\right) \text { and } k_{r} \leq i_{r}, \\ 0 \text { otherwise. }\end{array}\right.$

Proof - To expand a product $V_{I}^{\prime} V_{J}^{\prime}$, we have to compute the saillance compositions $K$ of permutations $\nu$ occuring in the shifted shuffle of permutations $\sigma$ and $\tau$ of respective saillance compositions $I$ and $J$.

We shall first discuss the number of saillances of $\nu$. The subword containing the $q \in[p, p+r]$ values of the saillances of $\nu$ consists of a prefix of the saillances of $\sigma$ followed by all shifted saillances of $\tau$. So, following the notations of the theorem, $q \in[p, p+r]$.

Now, if $q=p$, the saillances are exactly those of $\tau$ so this case corresponds to permutations $\nu$ such that $\nu_{1}=\tau_{1}+m$. In that case, between two saillances of $\nu$, there must be at least the corresponding values in $\tau$ and some values belonging to $\sigma$, hence the product of binomial coefficients since these values were shuffled in all possible ways with values of $\sigma$.

If $r=q-p>0$, the first $r-1$ saillances correspond to the saillances of $\sigma$, which means in particular that the value $\tau_{1}+m$ cannot appear too early, so that, the first $(r-1)$ parts of $K$ have to be equal to the first $(r-1)$ parts of $I$. The next part of $K$ has to satisfy $k_{r}<i_{r}$, meaning that either we meet $\tau_{1}+m$ or the next saillance of $\sigma$. And now the same explanation as before applies: each saillance value in $\nu$ coming from $\tau$ must be at least as far apart as these values in $\tau$, since they were shuffled with values of $\sigma$, whence the product of binomial coefficients.

For example, let us compute the coefficient of $V_{51}^{\prime}$ in $V_{2}^{\prime} V_{31}^{\prime}$.

This amounts to computing the number of permutations in 21 U $3124=21$ Ш 5346 with saillances at positions $\{1,6\}$. In that case, the first value has to be 5 and the last one has to be 6 , all remaining values being at any possible place, hence $\left(\begin{array}{l}4 \\ 2\end{array}\right)$ : put 21 and 34 in any order in the middle positions.

Let us now compute the coefficient of $V_{2131}^{\prime}$ in $V_{21}^{\prime} V_{121}^{\prime}$.

This amounts to computing the number of permutations in $213 \uplus 1324=213 \amalg 4657$ of saillances at positions $\{1,3,4,7\}$. Since there are four saillances, the first one has to be the 2 and the others are 4, 6, and 7 . So we need 2 in position 1 , then 4 in position 3, then 6 in position 4 , and 7 in position 7 . The value 5 of 4657 is in position 5 or 6 (between values 6 and 7 ), value 1 has to be in position 2 (no saillance there) and value 3 in position either 5 or 6 , hence the binomial coefficient $\left(\begin{array}{l}2 \\ 1\end{array}\right)$ showing that values 3 and 5 were shuffled together at positions 5 and 6 . 
For example,

$$
\begin{aligned}
V_{2}^{\prime} V_{31}^{\prime} & =V_{132}^{\prime}+3 V_{141}^{\prime}+V_{231}^{\prime}+V_{33}^{\prime}+3 V_{42}^{\prime}+6 V_{51}^{\prime} \\
V_{31}^{\prime} V_{2}^{\prime} & =4 V_{15}^{\prime}+3 V_{24}^{\prime}+V_{312}^{\prime}+2 V_{33}^{\prime}+5 V_{6}^{\prime} \\
V_{21}^{\prime} V_{121}^{\prime} & =V_{1123}^{\prime}+2 V_{1132}^{\prime}+3 V_{1141}^{\prime}+V_{1222}^{\prime}+2 V_{1231}^{\prime}+V_{124}^{\prime}+V_{1321}^{\prime} \\
& +2 V_{133}^{\prime}+3 V_{142}^{\prime}+4 V_{151}^{\prime}+V_{21121}^{\prime}+V_{2122}^{\prime}+2 V_{2131}^{\prime}+V_{2221}^{\prime}+V_{223}^{\prime} \\
& +2 V_{232}^{\prime}+3 V_{241}^{\prime}+V_{322}^{\prime}+2 V_{331}^{\prime}+V_{421}^{\prime}
\end{aligned}
$$

The only known basis which appears to be related to $V_{I}$ is the $\Psi$ basis:

Proposition 5.6. The $V$-expansion of $\Psi_{n}$ is

$$
\Psi_{n}=\sum_{I \models n}(-1)^{\ell(I)-1} V_{I} .
$$

Proof - We know that $\Psi_{n}=\sum_{k=0}^{n} R_{1^{k}, n-k}$, and we have in fact

$$
R_{1^{k}, n-k}=\sum_{\ell(I)=k+1, I \models n} V_{I} .
$$

Indeed, the sum of the permutations whose recoils are exactly $1,2, \ldots, k$ is $(k+$ 1) $(k \cdots 21 \omega k+2 \cdots n)$, and the saillance compositions of these permutations are precisely all the compositions of $n$ of length $k+1$.

\section{ApPENDix: insertion ALGORITHMS FOR SOME PATTERN-REPLACEMENT RELATIONS}

This appendix provides the proof of Proposition 5.1, together with some supplementary material, answering a question asked by Darij Grinberg [7]: to explain why the cardinalities of the equivalence classes of two relations considered in [12] have the same distribution. One of these relations turns out to be, up to mirror image of the patterns, the one inducing equality of the saillance compositions on the inverse permutations.

6.1. Background. A pattern-replacement relation is an equivalence relation on permutations defined by sets of patterns of the same size, two permutations being equivalent if one is obtained from the other by rearranging the letters forming a pattern of the set so as to form another one from the same set. Historically, the first example was defined by the two sets $\{132,312\}$ and $\{213,231\}$ : this is the well-known Knuth equivalence, whose classes are the fibers of the Robinson-Schensted correspondence.

Actually, the Knuth relations are defined on words over a totally ordered alphabet, and the quotient of the free monoid by the congruence generated by these relations is the celebrated plactic monoid, which has been for many years considered as a unique and singular object. It was the discovery of quantum groups and crystal bases which led to the understanding that the plactic monoid was associated with the root systems of type $A$, and that such objects existed for other types [14, 15]. 
This was however not the end of the story, as the investigation of the representationtheoretical meaning of quasi-symmetric functions led to the discovery of the hypoplactic monoid, which, while related in some way to quantum groups, does not fit in the previous pattern [11].

Finally, the investigation of the product rule of the Loday-Ronco Hopf algebra of planar binary trees led to the sylvester monoid, and other combinatorial Hopf algebras provided many new examples, for which no representation theoretical interpretation is known [8].

All these monoids induce pattern-replacement equivalences when restricted to permutations. These equivalences are rather special, in that they are induced by congruences on words which are compatible with standardization and restrictions to intervals, two properties ensuring that they can be used to define Hopf algebras [22, 21]. Also, in each of these cases, there is an insertion algorithm analogous to the Robinson-Schensted correspondence.

One may therefore wonder whether there are other pattern-replacement equivalences, not necessarily coming from such congruences, for which there is still an insertion algorithm, and some other interesting properties such as a closed formula for the number of classes, or for the cardinality of a class.

These last two points have been thoroughly investigated in the recent papers [12, 13, 5, 16, 23, 26]. In this appendix, we provide insertion algorithms for two examples from [12]. Both can be extended to bijections by introducing a $Q$-symbol, which turns out to be in both cases increasing trees of a special shape, thus explaining the curious fact that both equivalences have the same distribution of the cardinalities of classes.

6.2. The $\{\{312,321\},\{123,132\}\}$-equivalence. Consider the equivalence $\equiv$ generated by the following relations [12, Section 3.1]: for $a<b<c$,

$$
\begin{aligned}
& c a b \equiv c b a \\
& a b c \equiv a c b .
\end{aligned}
$$

We shall define an algorithm sending a permutation to a poset, whose Hasse diagram will be a labeled tree of a special shape. First, define the $W$-chain of $\sigma=\sigma_{1} \ldots \sigma_{n}$ as the sequence $S=\left(s_{1}, \ldots, s_{k}\right)$ such that $s_{k}$ is the position of whichever is rightmost between 1 and $n$. Any other $s_{i}$ satisfies that $\sigma_{1} \ldots \sigma_{s_{i+1}}$ has $\sigma_{s_{i}}$ as an extremum (the other one being $\left.\sigma_{s_{i+1}}\right)$. Note that this definition coincides essentially with the $W_{w}$ sets of Definition 3.3 of [12].

Now, given a W-chain, represent it as a chain poset with $\sigma_{s_{1}}$ at the top and $\sigma_{s_{k}}$ at the bottom, and place all other values $\sigma_{j}$ of $\sigma$ as leaves of the topmost element of the chain $\sigma_{s_{i}}$ (regarded as a linear tree) such that $\sigma_{j}$ belongs to the interval between $\sigma_{s_{i}}$ and $\sigma_{s_{i+1}}$. Denote the result by $P(\sigma)$.

For example, with $\sigma=532498617$, the $W$-chain is $[1,3,5,8]$ and the corresponding values are $[5,2,9,1]$. Now, 3 and 4 end up as leaves of 5 , and all remaining values as leaves of 2 . 


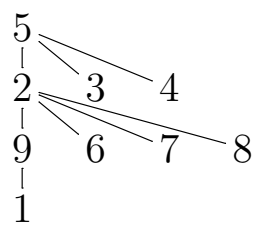

One can extend this algorithm to a bijection by memorizing in a second tree of the same shape the position of letter $i$ in $\sigma$. It follows from the definition of the chain that this yields an increasing tree, that we shall denote by $Q(\sigma)$.

In our example, $Q(\sigma)$ is

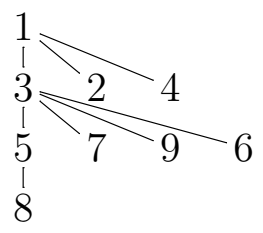

Theorem 6.1. Consider a permutation $\sigma$. The linear extensions of $P(\sigma)$, which are in bijection with the increasing trees of the same shape as $P(\sigma)$, are exactly the permutations $\equiv$-equivalent to $\sigma$.

Proof - All the necessary material is present in Kuszmaul's paper [12: he proves in Lemma 3.4 that if $w \equiv w^{\prime}$ then they have same $W$-set hence same poset. And he also proves that if $w$ and $w^{\prime}$ have same chain, they have same origin permutation (Lemma 3.6) and that any permutation is equivalent to its origin permutation (Lemma 3.7).

Now, given this property, one recovers instantly all the results of [12] concerning this pattern.

- There are $2^{n-1}$ classes of permutations of size $n$. Indeed, there are $2^{n-1}$ possible $W$-sets: given any subset $S$ of $[1, n]$ containing both 1 and $n$, there are two ways of ordering it as a chain: put either 1 or $n$ at the bottom and work your way up by taking alternatively the remaining maximum and minimum in $S$.

- The size of a class is given by an explicit hook-length formula: indeed, it is equal to the number of linear extensions of a poset whose Hasse diagram is a (very special) tree.

In our example, the hook-lengths are

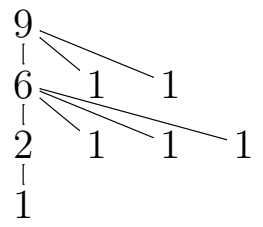

so that the cardinality of the class is $\frac{9 !}{9 \cdot 6 \cdot 1^{6}}=3360$.

The counting of equivalence classes can be easily refined as follows. 
Proposition 6.2. Among the $2^{n-1}$ classes, there are exactly $\left(\begin{array}{l}n-1 \\ k-1\end{array}\right)$ classes of permutations beginning with letter $k$.

Proof - This amounts to counting (e.g., by induction) $W$-sets beginning with $k$. More precisely, if one denotes by $A_{n}^{k}$ the number of such classes and $A_{n}^{k^{+}}$(resp. $A_{n}^{k^{-}}$) the number of such classes whose second value of the chain is greater (resp. smaller) than the first, there are exactly $\left(\begin{array}{l}n-2 \\ k-1\end{array}\right)$ (resp. $\left.\left(\begin{array}{l}n-2 \\ k-2\end{array}\right)\right)$ such classes.

6.3. The $\{\{123,132\},\{213,231\}\}$-equivalence. Consider the equivalence $\equiv_{2}$ generated by the relations [12, Section 3.11]: for $a<b<c$,

$$
\begin{aligned}
& b a c \equiv_{2} b c a \\
& a b c \equiv_{2} a c b .
\end{aligned}
$$

Define the left-to-right minima, $\operatorname{lrm}$ for short of $\sigma=\sigma_{1} \ldots \sigma_{n}$ as the sequence $S=\left\{s_{1}, \ldots, s_{k}\right\}$ such that $s_{i}$ is the $i$-th smallest integer such that $\sigma_{s_{i}}$ has only greater values to its left in $\sigma$.

Proposition 6.3. The $\equiv_{2}$ classes are exactly the sets of permutations having the same left-to-right minima.

Proof - Following [12], one first checks that in any $\equiv_{2}$ class, there is exactly one $V$ permutation (as defined in [12]) which is, by the way, the lexicographically smallest element of the class. This time, the proof is very easy since one can orient the relations (which is equivalent to saying that the cardinality of the classes are obtained by pattern avoidance, hence relating to Theorem 4.7 of [12]) and decide to rewrite any pattern $a c b$ into $a b c$ and any pattern $b c a$ into $b a c$. The words having neither $a c b$ nor $b c a$ patterns are $V$-permutations. This proves that any class has at least one such element. Conversely, it is obvious that the lrm of a permutation does not change with any rewriting, hence there cannot be two $V$-permutations in the same class.

Let us now define an algorithm sending a permutation to a poset. Given the lrm, represent it as a chain poset (again, regarded as a linear tree) with $\sigma_{s_{1}}$ at the top and $\sigma_{s_{k}}$ at its bottom, and place all other values $\sigma_{j}$ of $\sigma$ as leaves of the topmost element of the chain that is smaller than $\sigma_{j}$. Denote the result by $P_{2}(\sigma)$.

For example, with $\sigma=739465281$, its $\operatorname{lrm}$ is $[1,2,7,9]$ and the corresponding values are $[7,3,2,1]$. Now, 8 and 9 end as leaves of 7 , and all remaining values as leaves of 3.

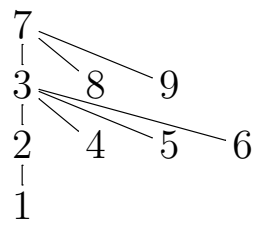


Again, this algorithm can be extended to a bijection by memorizing in a second tree of the same shape as the first one, where letter $i$ appears in $\sigma$. By the definition of the chain, this yields an increasing tree, that we shall denote by $Q_{2}(\sigma)$.

In our example, $Q_{2}(\sigma)$ is

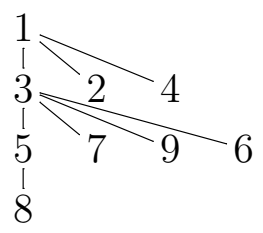

Theorem 6.4. Consider a permutation $\sigma$. All linear extensions of $P_{2}(\sigma)$, which are in bijection with the increasing trees of the same shape as $P_{2}(\sigma)$, are exactly the permutations equivalent to $\sigma$ under $\equiv_{2}$.

Proof - It is obvious that two permutations having the same lrm give the same result by the $P_{2}$ algorithm. Conversely, consider $P_{2}(\sigma)$. Given the description of the poset, it is direct that all its linear extensions have same $1 \mathrm{rm}$.

Note that given a naked poset, there are two ways to label it: consider the longest chain in it and label the last two elements either 1 and 2 or 2 and 1 . This being fixed, all the other vertices have no choice for their labeling.

Corollary 6.5 (Prop. 3.11 of [12]). The multisets of sizes of classes in $S_{n}$ under both equivalences are the same.

Proof - Both equivalences $\equiv$ and $\equiv_{2}$ give rise to the same naked posets and all posets have, in each case, exactly two different labelings.

Finally, it is pretty clear that one could transform these equalities into a bijection between classes, e.g., sending a poset of $\equiv$ with 1 below $n$ to the poset of $\equiv_{2}$ of the same shape with 1 below 2, and then extending this bijection to permutations by reading the same linear extensions out of these (in other words, the one-to-one correspondence relates permutations having same $Q$-symbols).

6.4. The $\{\{321,231\},\{312,132\}\}$-equivalence. These relations are the mirror-image of those defining $\equiv_{2}$, which transforms the equivalence relation on the inverse permutations into that of having the same left-to right maxima, that is, the same saillance composition, whence Proposition 5.1 .

\section{References}

[1] C. Berg, N. Bergeron, F. Saliola, L. Serrano, and M. Zabrocki, A lift of the Schur and Hall-Littlewood bases to non-commutative symmetric functions, Canad. J. Math. 66 (2014), $525-565$.

[2] A. BJÖRNER and M. WACHS, q-hook-length formulas for forests, J. Combinatorial Theory, Ser. A 52 (1989), 165-187.

[3] L. CARlitz, Generalized Stirling numbers, Combinatorial Analysis Notes, Duke University, 1968, 1-7. 
[4] G. Duchamp, F. Hivert, and J.-Y. Thibon, Noncommutative symmetric functions VI: free quasi-symmetric functions and related algebras, Internat. J. Algebra and Computation 12 (2002), 671-717.

[5] V. Fazel-Rezai, Equivalence Classes of Permutations Modulo Replacements Between 123 and Two-Integer Patterns. arXiv:1309.4802, 2013.

[6] I. M. Gelfand, D. Krob, A. Lascoux, B. Leclerc, V. S. Retakh, and J.-Y. Thibon, Noncommutative symmetric functions, Adv. Math. 112, 1995, 218-348.

[7] D. Grinberg, Private communication, $201 \%$.

[8] F. Hivert, J.-C. Novelli, and J.-Y. Thibon, The algebra of binary search trees, Theoretical Computer Science 339 (2005), 129-165.

[9] F. Hivert, J.-C. Novelli, L. Tevlin, and J.-Y. Thibon, Permutation statistics related to a class of noncommutative symmetric functions and generalizations of the Genocchi numbers, Selecta Math. (N.S.) 15 (2009), no. 1, 105-119.

[10] D. Krob, B. Leclerc, and J.-Y. Thibon, Noncommutative symmetric functions II: Transformations of alphabets, Intern. J. Alg. Comput. 7 no. 2, (1997), 181-264.

[11] D. Krob and J.-Y.Thiвon, Noncommutative symmetric functions IV: Quantum linear groups and Hecke algebras at $q=0$, J. Alg. Comb. 6 (1997), 339-376.

[12] W. Kuszmaul, Counting Permutations Modulo Pattern-Replacement Equivalences for ThreeLetter Patterns, Electronic Journal of Combinatorics 20 (4) (2013), \#P10. arXiv:1304.5667v2.

[13] W. Kuszmaul and Z. Zhou, Equivalence Classes in $S_{n}$ for Three Families of PatternReplacement Relations, MIT PRIMES, 2013. http://web.mit.edu/primes/materials/2012/Kuszmaul-Zhou.pdf.

[14] A. Lascoux, B. Leclerc, and J.-Y. ThiBon, Crystal graphs and q-analogues of weight multiplicities for the root system $A_{n}$, Lett. Math. Phys. 35 (1995),359-374.

[15] P. Littelmann, A plactic algebra for semisimple Lie algebras, Adv. Math., 124 (1996), 312331.

[16] S. Linton, J. Propp, T. Roby, and J. West, Equivalence Relations of Permutations Generated by Constrained Transpositions. DMTCS Proceedings, North America, July 2010. http://www.dmtcs.org/dmtcs-ojs/index.php/proceedings/article/view/dmAN0168.

[17] J.-L. LoDAY, Exponential series without denominators, Lie Theory and its Applications in Physics, IX International Workshop 2013, $<10.1007 / 978-4-431-54270-4 \_7>$. <hal-01267260>.

[18] M. Lothaire, Combinatorics on Words, 2nd Ed., Cambridge University press, 1997.

[19] I. G. Macdonald, Symmetric functions and Hall polynomials, 2nd ed., Clarendon Press, Oxford 1995.

[20] J.-C. Novelli, J.-Y. Thibon, and F. Toumazet, Noncommutative Bell polynomials and the dual immaculate basis, arXiv:1705.08113.

[21] J.-C. Novelli and J.-Y. Thibon, Hopf Algebras of $m$-permutations, $(m+1)$-ary trees, and $m$-parking functions, arXiv:1403.5962.

[22] J. Nzeutchap, Correspondances de Schensted-Fomin, Algèbres de Hopf et graphes gradués en dualité, Thèse de Doctorat, Université de Rouen, 2008.

[23] A. Pierrot, D. Rossin, and J. West, Adjacent transformations in permutations. FPSAC 2011 Proceedings, Discrete Math. Theor. Comput. Sci. Proc., 2011.

http: //www.dmtcs.org/dmtcs-ojs/index.php/proceedings/article/view/dmA00167/3638.

[24] R. Schimming and S. Z. RIDA, Noncommutative Bell polynomials, Internat. J. Algebra Comput. 6 (1996), 635-644.

[25] The On-Line Encyclopedia of Integer Sequences, published electronically at http://oeis.org, 2010.

[26] R. Stanley, An equivalence relation on the symmetric group and multiplicity-free flag $h$ vectors. arXiv:1208.3540, 2012.

[27] J.-Y. Thibon, Lectures on Noncommutative Symmetric Functions, Memoirs of the Japan Mathematical Society 11 (2001), 39-94. 


\section{TABLES}

$$
\begin{aligned}
& U_{2}=F_{2} \\
& U_{11}=F_{11} \\
& U_{3}=F_{3} \\
& U_{21}=F_{12}+F_{21} \\
& U_{12}=F_{12} \\
& U_{111}=F_{111}+F_{21} \\
& U_{4}=F_{4} \\
& U_{31}=F_{13}+F_{22}+F_{31} \\
& U_{22}=F_{13}+F_{22} \\
& U_{211}=F_{112}+2 F_{121}+F_{211}+F_{22}+F_{31} \\
& U_{13}=F_{13} \\
& U_{121}=F_{112}+F_{121}+F_{22} \\
& U_{112}=F_{112}+F_{22} \\
& U_{1111}=F_{1111}+2 F_{121}+2 F_{211}+F_{31} \\
& U_{5}=F_{5} \\
& U_{41}=F_{14}+F_{23}+F_{32}+F_{41} \\
& U_{32}=F_{14}+F_{23}+F_{32} \\
& U_{311}=F_{113}+2 F_{122}+2 F_{131}+F_{212}+2 F_{221}+F_{23}+F_{311}+F_{32}+F_{41} \\
& U_{23}=F_{14}+F_{23} \\
& U_{221}=F_{113}+2 F_{122}+F_{131}+F_{212}+F_{221}+F_{23}+F_{32} \\
& U_{212}=F_{113}+2 F_{122}+F_{212}+F_{23}+F_{32} \\
& U_{2111}=F_{1112}+3 F_{1121}+3 F_{1211}+2 F_{122}+3 F_{131}+F_{2111}+2 F_{212}+5 F_{221}+2 F_{311}+F_{32}+F_{41} \\
& U_{14}=F_{14} \\
& U_{131}=F_{113}+F_{122}+F_{131}+F_{23} \\
& U_{122}=F_{113}+F_{122}+F_{23} \\
& U_{1211}=F_{1112}+2 F_{1121}+F_{1211}+2 F_{122}+F_{131}+2 F_{212}+2 F_{221}+F_{32} \\
& U_{113}=F_{113}+F_{23} \\
& U_{1121}=F_{1112}+F_{1121}+2 F_{122}+2 F_{212}+F_{221}+F_{32} \\
& U_{1112}=F_{1112}+2 F_{122}+2 F_{212}+F_{32} \\
& U_{11111}=F_{11111}+3 F_{1121}+5 F_{1211}+3 F_{131}+3 F_{2111}+5 F_{221}+3 F_{311}+F_{41}
\end{aligned}
$$

Figure 3 . The basis $U_{I}$ for $n \leq 5$. 


$$
\begin{aligned}
& \left(\begin{array}{llll}
3 & 12 & 21 & 111 \\
1 & 0 & 0 & 0 \\
0 & 1 & 1 & 0 \\
0 & 0 & 1 & 1 \\
0 & 0 & 0 & 1
\end{array}\right) \\
& \begin{array}{llllllll}
4 & 13 & 22 & 31 & 112 & 121 & 211 & 1111
\end{array} \\
& \left(\begin{array}{llllllll}
1 & 0 & 0 & 0 & 0 & 0 & 0 & 0 \\
0 & 1 & 1 & 1 & 0 & 0 & 0 & 0 \\
0 & 0 & 1 & 1 & 1 & 1 & 1 & 0 \\
0 & 0 & 0 & 1 & 0 & 0 & 1 & 1 \\
0 & 0 & 0 & 0 & 1 & 1 & 1 & 0 \\
0 & 0 & 0 & 0 & 0 & 1 & 2 & 2 \\
0 & 0 & 0 & 0 & 0 & 0 & 1 & 2 \\
0 & 0 & 0 & 0 & 0 & 0 & 0 & 1
\end{array}\right)
\end{aligned}
$$

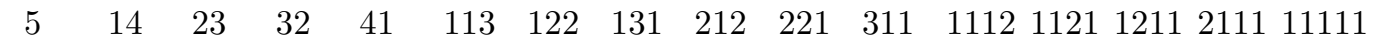

$$
\begin{aligned}
& \left(\begin{array}{cccccccccccccccc}
1 & 0 & 0 & 0 & 0 & 0 & 0 & 0 & 0 & 0 & 0 & 0 & 0 & 0 & 0 & 0 \\
0 & 1 & 1 & 1 & 1 & 0 & 0 & 0 & 0 & 0 & 0 & 0 & 0 & 0 & 0 & 0 \\
0 & 0 & 1 & 1 & 1 & 1 & 1 & 1 & 1 & 1 & 1 & 0 & 0 & 0 & 0 & 0 \\
0 & 0 & 0 & 1 & 1 & 0 & 0 & 0 & 1 & 1 & 1 & 1 & 1 & 1 & 1 & 0 \\
0 & 0 & 0 & 0 & 1 & 0 & 0 & 0 & 0 & 0 & 1 & 0 & 0 & 0 & 1 & 1 \\
0 & 0 & 0 & 0 & 0 & 1 & 1 & 1 & 1 & 1 & 1 & 0 & 0 & 0 & 0 & 0 \\
0 & 0 & 0 & 0 & 0 & 0 & 1 & 1 & 2 & 2 & 2 & 2 & 2 & 2 & 2 & 0 \\
0 & 0 & 0 & 0 & 0 & 0 & 0 & 1 & 0 & 1 & 2 & 0 & 0 & 1 & 3 & 3 \\
0 & 0 & 0 & 0 & 0 & 0 & 0 & 0 & 1 & 1 & 1 & 2 & 2 & 2 & 2 & 0 \\
0 & 0 & 0 & 0 & 0 & 0 & 0 & 0 & 0 & 1 & 2 & 0 & 1 & 2 & 5 & 5 \\
0 & 0 & 0 & 0 & 0 & 0 & 0 & 0 & 0 & 0 & 1 & 0 & 0 & 0 & 2 & 3 \\
0 & 0 & 0 & 0 & 0 & 0 & 0 & 0 & 0 & 0 & 0 & 1 & 1 & 1 & 1 & 0 \\
0 & 0 & 0 & 0 & 0 & 0 & 0 & 0 & 0 & 0 & 0 & 0 & 1 & 2 & 3 & 3 \\
0 & 0 & 0 & 0 & 0 & 0 & 0 & 0 & 0 & 0 & 0 & 0 & 0 & 1 & 3 & 5 \\
0 & 0 & 0 & 0 & 0 & 0 & 0 & 0 & 0 & 0 & 0 & 0 & 0 & 0 & 1 & 3 \\
0 & 0 & 0 & 0 & 0 & 0 & 0 & 0 & 0 & 0 & 0 & 0 & 0 & 0 & 0 & 1
\end{array}\right)
\end{aligned}
$$

FiguRE 4. Transition matrices $M_{n}$ for $n \leq 5$.

[Novelli, Thibon, Toumazet] Laboratoire d'informatique Gaspard-Monge, Université Paris-Est Marne-la-Vallée, 5, Boulevard Descartes, Champs-sur-Marne, 77454 Marne-la-Vallée Cedex 2, France

E-mail address, Jean-Christophe Novelli: novelli@u-pem.fr

E-mail address, Jean-Yves Thibon: jyt@u-pem.fr

E-mail address, Frédéric Toumazet: frederic.toumazet@u-pem.fr 


$$
\begin{aligned}
& V_{2}=R_{2} \\
& V_{11}=R_{11} \\
& V_{3}=R_{3} \\
& V_{21}=-R_{111}+R_{21} \\
& V_{12}=R_{111}+R_{12}-R_{21} \\
& V_{111}=R_{111} \\
& V_{4}=R_{4} \\
& V_{31}=R_{1111}-R_{211}+R_{31} \\
& V_{22}=-R_{1111}-R_{112}+R_{211}+R_{22}-R_{31} \\
& V_{211}=-2 R_{1111}+R_{211} \\
& V_{13}=R_{112}+R_{13}-R_{22} \\
& V_{121}=2 R_{1111}+R_{121}-2 R_{211} \\
& V_{112}=R_{112}-R_{121}+R_{211} \\
& V_{1111}=R_{1111} \\
& V_{5}=R_{5} \\
& V_{41}=-R_{11111}+R_{2111}-R_{311}+R_{41} \\
& V_{32}=R_{11111}+R_{1112}-R_{2111}-R_{212}+R_{311}+R_{32}-R_{41} \\
& V_{311}=3 R_{11111}-2 R_{2111}+R_{311} \\
& V_{23}=-R_{1112}-R_{113}+R_{212}+R_{23}-R_{32} \\
& V_{221}=-2 R_{11111}-R_{1121}+2 R_{2111}+R_{221}-2 R_{311} \\
& V_{212}=-R_{11111}-2 R_{1112}+R_{1121}+R_{212}-R_{221}+R_{311} \\
& V_{2111}=-3 R_{11111}+R_{2111} \\
& V_{14}=R_{113}+R_{14}-R_{23} \\
& V_{131}=-2 R_{11111}+R_{1121}-R_{1211}+R_{131}+2 R_{2111}-R_{221} \\
& V_{122}=2 R_{11111}+2 R_{1112}-R_{1121}+R_{1211}+R_{122}-R_{131}-2 R_{2111}-2 R_{212}+R_{221} \\
& V_{1211}=4 R_{11111}+R_{1211}-3 R_{2111} \\
& V_{113}=R_{113}-R_{122}+R_{212} \\
& V_{1121}=-2 R_{11111}+R_{1121}-2 R_{1211}+3 R_{2111} \\
& V_{1112}=R_{11111}+R_{1112}-R_{1121}+R_{1211}-R_{2111} \\
& V_{11111}=R_{11111}
\end{aligned}
$$

Figure 5. The basis $V_{I}$ for $n \leq 5$. 


$$
\begin{aligned}
& V_{2}=\Lambda_{11}-\Lambda_{2} \\
& V_{11}=\Lambda_{2} \\
& V_{3}=\Lambda_{111}-\Lambda_{12}-\Lambda_{21}+\Lambda_{3} \\
& V_{21}=\Lambda_{12}-2 \Lambda_{3} \\
& V_{12}=-\Lambda_{12}+\Lambda_{21}+\Lambda_{3} \\
& V_{111}=\Lambda_{3} \\
& V_{4}=\Lambda_{1111}-\Lambda_{112}-\Lambda_{121}+\Lambda_{13}-\Lambda_{211}+\Lambda_{22}+\Lambda_{31}-\Lambda_{4} \\
& V_{31}=\Lambda_{112}-2 \Lambda_{13}-\Lambda_{22}+3 \Lambda_{4} \\
& V_{22}=-\Lambda_{112}+\Lambda_{121}+\Lambda_{13}+\Lambda_{22}-2 \Lambda_{31}-\Lambda_{4} \\
& V_{211}=\Lambda_{13}-3 \Lambda_{4} \\
& V_{13}=-\Lambda_{121}+\Lambda_{13}+\Lambda_{211}-\Lambda_{22}+\Lambda_{31}-\Lambda_{4} \\
& V_{121}=-2 \Lambda_{13}+\Lambda_{22}+3 \Lambda_{4} \\
& V_{112}=\Lambda_{13}-\Lambda_{22}+\Lambda_{31}-\Lambda_{4} \\
& V_{1111}=\Lambda_{4} \\
& \begin{aligned}
V_{5} & =\Lambda_{11111}-\Lambda_{1112}-\Lambda_{1121}+\Lambda_{113}-\Lambda_{1211}+\Lambda_{122} \\
& +\Lambda_{131}-\Lambda_{14}-\Lambda_{2111}+\Lambda_{212}+\Lambda_{221}-\Lambda_{23}+\Lambda_{311}-\Lambda_{32}-\Lambda_{41}+\Lambda_{5} \\
V_{41} & =\Lambda_{1112}-2 \Lambda_{113}-\Lambda_{122}+3 \Lambda_{14}-\Lambda_{212}+2 \Lambda_{23}+\Lambda_{32}-4 \Lambda_{5} \\
V_{32} & =-\Lambda_{1112}+\Lambda_{1121}+\Lambda_{113}+\Lambda_{122}-2 \Lambda_{131}-\Lambda_{14}+\Lambda_{212}-\Lambda_{221}-\Lambda_{23}-\Lambda_{32}+3 \Lambda_{41}+\Lambda_{5} \\
V_{311} & =\Lambda_{113}-3 \Lambda_{14}-\Lambda_{23}+6 \Lambda_{5} \\
V_{23} & =-\Lambda_{1121}+\Lambda_{113}+\Lambda_{1211}-\Lambda_{122}+\Lambda_{131}-\Lambda_{14}+\Lambda_{221}-\Lambda_{23}-2 \Lambda_{311}+2 \Lambda_{32}-\Lambda_{41}+\Lambda_{5} \\
V_{221} & =-2 \Lambda_{113}+\Lambda_{122}+3 \Lambda_{14}+2 \Lambda_{23}-2 \Lambda_{32}-4 \Lambda_{5} \\
V_{212} & =\Lambda_{113}-\Lambda_{122}+\Lambda_{131}-\Lambda_{14}-\Lambda_{23}+2 \Lambda_{32}-3 \Lambda_{41}+\Lambda_{5} \\
V_{2111} & =\Lambda_{14}-4 \Lambda_{5} \\
V_{14} & =-\Lambda_{1211}+\Lambda_{122}+\Lambda_{131}-\Lambda_{14}+\Lambda_{2111}-\Lambda_{212}-\Lambda_{221}+\Lambda_{23}+\Lambda_{311}-\Lambda_{32}-\Lambda_{41}+\Lambda_{5} \\
V_{131} & =-\Lambda_{122}+3 \Lambda_{14}+\Lambda_{212}-2 \Lambda_{23}+\Lambda_{32}-4 \Lambda_{5} \\
V_{122} & =\Lambda_{122}-2 \Lambda_{131}-\Lambda_{14}-\Lambda_{212}+\Lambda_{221}+\Lambda_{23}-\Lambda_{32}+3 \Lambda_{41}+\Lambda_{5} \\
V_{1211} & =-3 \Lambda_{14}+\Lambda_{23}+6 \Lambda_{5} \\
V_{113} & =\Lambda_{131}-\Lambda_{14}-\Lambda_{221}+\Lambda_{23}+\Lambda_{311}-\Lambda_{32}-\Lambda_{41}+\Lambda_{5} \\
V_{1121} & =3 \Lambda_{14}-2 \Lambda_{23}+\Lambda_{32}-4 \Lambda_{5} \\
V_{1112} & =-\Lambda_{14}+\Lambda_{23}-\Lambda_{32}+\Lambda_{41}+\Lambda_{5} \\
V_{11111} & =\Lambda_{5}
\end{aligned}
\end{aligned}
$$

Figure 6 . The basis $V_{I}$ on the $\Lambda^{J}$ for $n \leq 5$. 


$$
\begin{aligned}
& 211 \\
& \left(\begin{array}{ll}
1 & 0 \\
0 & 1
\end{array}\right) \\
& \begin{array}{llll}
3 & 12 & 21 & 111
\end{array} \\
& \left(\begin{array}{rrrr}
1 & 0 & 0 & 0 \\
0 & 1 & -1 & 1 \\
0 & 0 & 1 & -1 \\
0 & 0 & 0 & 1
\end{array}\right) \\
& \begin{array}{llllllll}
4 & 13 & 22 & 31 & 112 & 121 & 211 & 1111
\end{array} \\
& \left(\begin{array}{llllllll}
1 & 0 & 0 & 0 & 0 & 0 & 0 & 0 \\
0 & 1 & -1 & 0 & 1 & 0 & 0 & 0 \\
0 & 0 & 1 & -1 & -1 & 0 & 1 & -1 \\
0 & 0 & 0 & 1 & 0 & 0 & -1 & 1 \\
0 & 0 & 0 & 0 & 1 & -1 & 1 & 0 \\
0 & 0 & 0 & 0 & 0 & 1 & -2 & 2 \\
0 & 0 & 0 & 0 & 0 & 0 & 1 & -2 \\
0 & 0 & 0 & 0 & 0 & 0 & 0 & 1
\end{array}\right)
\end{aligned}
$$$$
\left(\begin{array}{llllllllllllllll}
5 & 14 & 23 & 32 & 41 & 113 & 122 & 131 & 212 & 221 & 311 & 1112 & 1121 & 1211 & 2111 & 1111 \\
1 & 0 & 0 & 0 & 0 & 0 & 0 & 0 & 0 & 0 & 0 & 0 & 0 & 0 & 0 & 0 \\
0 & 1 & -1 & 0 & 0 & 1 & 0 & 0 & 0 & 0 & 0 & 0 & 0 & 0 & 0 & 0 \\
0 & 0 & 1 & -1 & 0 & -1 & 0 & 0 & 1 & 0 & 0 & -1 & 0 & 0 & 0 & 0 \\
0 & 0 & 0 & 1 & -1 & 0 & 0 & 0 & -1 & 0 & 1 & 1 & 0 & 0 & -1 & 1 \\
0 & 0 & 0 & 0 & 1 & 0 & 0 & 0 & 0 & 0 & -1 & 0 & 0 & 0 & 1 & -1 \\
0 & 0 & 0 & 0 & 0 & 1 & -1 & 0 & 1 & 0 & 0 & 0 & 0 & 0 & 0 & 0 \\
0 & 0 & 0 & 0 & 0 & 0 & 1 & -1 & -2 & 1 & 0 & 2 & -1 & 1 & -2 & 2 \\
0 & 0 & 0 & 0 & 0 & 0 & 0 & 1 & 0 & -1 & 0 & 0 & 1 & -1 & 2 & -2 \\
0 & 0 & 0 & 0 & 0 & 0 & 0 & 0 & 1 & -1 & 1 & -2 & 1 & 0 & 0 & -1 \\
0 & 0 & 0 & 0 & 0 & 0 & 0 & 0 & 0 & 1 & -2 & 0 & -1 & 0 & 2 & -2 \\
0 & 0 & 0 & 0 & 0 & 0 & 0 & 0 & 0 & 0 & 1 & 0 & 0 & 0 & -2 & 3 \\
0 & 0 & 0 & 0 & 0 & 0 & 0 & 0 & 0 & 0 & 0 & 1 & -1 & 1 & -1 & 1 \\
0 & 0 & 0 & 0 & 0 & 0 & 0 & 0 & 0 & 0 & 0 & 0 & 1 & -2 & 3 & -2 \\
0 & 0 & 0 & 0 & 0 & 0 & 0 & 0 & 0 & 0 & 0 & 0 & 0 & 1 & -3 & 4 \\
0 & 0 & 0 & 0 & 0 & 0 & 0 & 0 & 0 & 0 & 0 & 0 & 0 & 0 & 1 & -3 \\
0 & 0 & 0 & 0 & 0 & 0 & 0 & 0 & 0 & 0 & 0 & 0 & 0 & 0 & 0 & 1
\end{array}\right)
$$

Figure 7 . The matrices $M_{n}^{-1}$ for $n \leq 5$. 